\title{
SPREADING RATE OF VIRUS BETWEEN INCOMING AND OUTGOING LINKS OF A WEBSITE THROUGH AN INTUITIONISTIC FUZZY GRAPH
}

\author{
B. Praba ${ }^{1}$, G. Deepa ${ }^{2} \S$, V.M. Chandrasekaran ${ }^{3}$ \\ ${ }^{1} \mathrm{SSN}$ College of Engineering \\ Kalavakkam, Chennai, 603110, Tamilnadu, INDIA \\ ${ }^{2,3}$ School of Advanced Sciences \\ VIT University \\ Vellore, 632014, Tamilnadu, INDIA
}

\begin{abstract}
In this paper we analyzed the spreading rate of virus between incoming and outgoing links to a website. We have taken the website http://www.pantechsolutions.net/. This website is modeled as an intuitionistic fuzzy graph $G=(V, E, \mu, \gamma)$ by considering the navigation of the customers. In this intuitionistic fuzzy graph, the links are considered as vertices and the path of the links is considered as edges. The weightage of each edge are considered as number of visitors (membership value), number of non-visitors (non-membership) and drop off case (intuitionistic fuzzy index). For four different time periods, the four links 1. Microcontroller-boards, 2. /Log-in html, 3. / and 4. /Project kits of the given website are taken for our calculation. In this intuitionistic fuzzy graph we constructed two intuitionistic fuzzy matrices using incoming and outgoing links to $G$. The energy of these matrices along with its lower and upper bound are discussed. The spreading rate of virus of the given graph in terms of these two matrices is discussed. These concepts are illustrated with real time example.
\end{abstract}

AMS Subject Classification: 05C72, 05C69, 05C22

Key Words: energy of a graph, energy of a fuzzy graph, energy of an intuitionistic fuzzy graph

Received: $\quad$ May 14, 2016

Revised: $\quad$ August 17, 2016

Published: October 6, 2016

${ }^{\S}$ Correspondence author (c) 2016 Academic Publications, Ltd. url: www.acadpubl.eu 


\section{Introduction}

Graph theory was introduced by Leonhard Euler when he solved the "Konigsberg bridge" problem in 1735. Many real life problems can be represented by graph. In computer science, graphs are used to represent networks of communications, data organization, computational devices, the flow of computation etc. The link structure of a website can be represented by a directed graph in which the vertices are the web pages available at the website and a directed edge from page $\mathrm{A}$ to page $\mathrm{B}$ exists if and only if $\mathrm{A}$ contains a link to $\mathrm{B}$ (see [11]). A similar approach can be taken to problems in travel, biology, computer chip design and many other fields. Hence graph theory is widely used in solving real time problems. But when the system is large and complex it is difficult to extract the exact information about the system using the classical graph theory. In such cases fuzzy graph is used to analyze the system. Fuzzy graph was introduced by Kaufmann in 1973 (see [8]). Intuitionistic fuzzy graph was introduced by Atanassov in 1999 (see [2]). Gutman introduced the concept of energy of a graph in 1978 (see [7]). The energy of a fuzzy graph and its bounds are discussed in 2013 (see [1]). The energy of an intuitionistic fuzzy graph and its bounds are discussed in 2014 (see [12]). The authors (see [5]) discussed the virus spread in an intuitionistic fuzzy network. The relationship between the number of visitors and the energy of an intuitionistic fuzzy graph is analyzed which is used to find the spreading rate of virus. Many authors (see [13], [3], [4], [9], [10]) discussed the existence of an epidemic threshold $\tau_{c}$. In 2015 (see [6]), Max-Min intuitionistic fuzzy matrix $M(G)$ of an intuitionistic fuzzy graph is defined and the extreme energy of $M(G)$ is obtained. Also an explicit expression of the coefficients of the characteristic polynomial of $M(G)$ is determined. These concepts are illustrated with real time example. This paper is organized as follows. In Section 2, we give all the basic definitions related to energy of an intuitionistic fuzzy graph. In Section 3, we defined the In-Min and Out-Min intuitionistic fuzzy matrix of an intuitionistic fuzzy graph. In Section 4, we illustrate these concepts by taking the website of http://www.pantechsolutions.net/. In Section 5, we give the conclusion. 


\section{Preliminaries}

\subsection{Intuitionistic Fuzzy Graph}

Definition 1. (see [12]) An intuitionistic fuzzy graph is defined as $G=$ $(V, E, \mu, \gamma)$ where $V$ is the set of vertices and $E$ is the set of edges. $\mu$ is a fuzzy membership function defined on $V \times V$ and $\gamma$ is a fuzzy non-membership function defined on $V \times V$. We denote $\mu\left(v_{i}, v_{j}\right)$ by $\mu_{i j}$ and $\gamma\left(v_{i}, v_{j}\right)$ by $\gamma_{i j}$ such that (i) $0 \leq \mu_{i j}+\gamma_{i j} \leq 1$ (ii) $0 \leq \mu_{i j}, \gamma_{i j}, \pi_{i j} \leq 1$, where $\pi_{i j}=1-\mu_{i j}-\gamma_{i j}$. Hence $(V \times V, \mu, \gamma)$ is an intuitionistic fuzzy set.

Definition 2. (see [12]) An intuitionistic fuzzy adjacency matrix of an intuitionistic fuzzy $G$ is defined as $A(I G)=\left[a_{i j}\right]$ where $a_{i j}=\left(\mu_{i j}, \gamma_{i j}\right)$. Note that $\mu_{i j}$ represents the strength of the relationship between $v_{i}$ and $v_{j}$ and $\gamma_{i j}$ represents the strength of the non-relationship between $v_{i}$ and $v_{j}$. The adjacency matrix of an intuitionistic fuzzy graph can be written as two matrices one containing the entries as membership values and the other containing the entries as non-membership values. i.e. $A(I G)=\left(\left(\mu_{i j}\right),\left(\gamma_{i j}\right)\right)$.

\subsection{Energy of an Intuitionistic Fuzzy Graph}

Definition 3. (see [12]) The eigen values of an intuitionistic fuzzy adjacency matrix $A(I G)$ is defined as $(X, Y)$ where $X$ is the set eigen values of $A\left(\mu_{i j}\right)$ and $Y$ is the set of eigen values of $A\left(\gamma_{i j}\right)$. The energy of an intuitionistic fuzzy graph $G=(V, E, \mu, \gamma)$ is defined as $\left(\sum_{\lambda_{i} \in X}\left|\lambda_{i}\right|, \sum_{\delta_{i} \in Y}\left|\delta_{i}\right|\right)$ where $\sum_{\lambda_{i} \in X}\left|\lambda_{i}\right|$ is defined as an energy of the membership matrix denoted by $E\left(\mu_{i j}(G)\right)$ and $\sum_{\delta_{i} \in Y}\left|\delta_{i}\right|$ is defined as an energy of the non-membership matrix denoted by $E\left(\gamma_{i j}(G)\right)$.

\subsection{Virus Spread in Intuitionistic Fuzzy Network}

Definition 4. (see [5]) If $E\left(\mu_{i j}(G)\right)>E\left(\gamma_{i j}(G)\right)$ i.e. If the number of visitors are maximum in an intuitionistic fuzzy network then the spreading rate of virus will be maximum. If $E\left(\mu_{i j}(G)\right)>E\left(\gamma_{i j}(G)\right)$ then the infection rate of an intuitionistic fuzzy network is defined as $\beta=\max _{i, j} \mu_{i j}$ and the curing rate of an intuitionistic fuzzy network is defined as $\delta=\min _{i, j} \gamma_{i j}$. The ratio $\tau=\frac{\beta}{\delta}(\delta \neq 0)$ is the effective spreading rate and $\tau_{c}=\frac{1}{\lambda_{\max } A\left(\mu_{i j}\right)}$ where $\lambda_{\max } A\left(\mu_{i j}\right)$ is the largest eigen value of the adjacency matrix $A\left(\mu_{i j}\right)$ of an intuitionistic fuzzy 
network.

Definition 5. (see [5]) If $E\left(\mu_{i j}(G)\right)<E\left(\gamma_{i j}(G)\right)$ i.e. If the number of visitors are minimum in an intuitionistic fuzzy network then the spreading rate of virus will be minimum. If $E\left(\mu_{i j}(G)\right)<E\left(\gamma_{i j}(G)\right)$ then the infection rate of an intuitionistic fuzzy network is defined as $\beta=\min _{i, j} \mu_{i j}$ and the curing rate of an intuitionistic fuzzy network is defined as $\delta=\max _{i, j} \gamma_{i j}$. The ratio $\tau=\frac{\beta}{\delta}(\delta \neq 0)$ is the effective spreading rate and $\tau_{c}=\frac{1}{\lambda_{\max } A\left(\gamma_{i j}\right)}$ where $\lambda_{\max } A\left(\gamma_{i j}\right)$ is the largest eigen value of the adjacency matrix $A\left(\gamma_{i j}\right)$ of an intuitionistic fuzzy network.

\section{Out Min-In Min Intuitionistic Fuzzy Matrix of an Intuitionistic Fuzzy Graph}

In this section, we consider an intuitionistic fuzzy graph and constructed two intuitionistic fuzzy matrices using incoming and outgoing links in this graph. The spreading rate of virus of the given graph is analyzed in terms these matrices.

Definition 6. Let $G=(V, E, \mu, \gamma)$ be an intuitionistic fuzzy graph. $\forall$ node $i$, define $\alpha_{j}=\max _{i} \mu_{i j}$ and $\sigma_{j}=\min _{i} \gamma_{i j}$.

Theorem 7. Let $G=(V, E, \mu, \gamma)$ be an intuitionistic fuzzy graph. If $\alpha_{j}=\max _{i} \mu_{i j}$ and $\sigma_{j}=\min _{i} \gamma_{i j}$ then $\left(\alpha_{j}, \sigma_{j}\right)$ is an intuitionistic fuzzy set on $V$.

Proof. If $\alpha_{j}=\max _{i} \mu_{i j}$ then $\alpha_{j}=\mu_{s j}$ for some $s \in V$. Similarly if $\sigma_{j}=$ $\min _{i} \gamma_{i j}$ then $\sigma_{j}=\gamma_{t j}$ for some $t \in V$. Here, we have $\gamma_{t j} \leq \gamma_{s j}$. That is $-\gamma_{t j} \geq$ ${ }^{i} \gamma_{s j}$. Now $\alpha_{j}=\mu_{s j} \leq 1-\gamma_{s j} \leq 1-\gamma_{t j}$. This gives $\mu_{s j}+\gamma_{t j} \leq 1$. That is $\alpha_{j}+\sigma_{j} \leq 1$. Therefore $0 \leq \alpha_{j}+\sigma_{j} \leq 1$. Hence $\left(\alpha_{j}, \sigma_{j}\right)$ is an intuitionistic fuzzy set on $V$.

Definition 8. Let $G=(V, E, \mu, \gamma)$ be an intuitionistic fuzzy graph. The In-Min intuitionistic fuzzy matrix of an intuitionistic fuzzy graph is defined as $M_{I}(G)=\left(\left(r_{i j}, s_{i j}\right)\right)$ where $r_{i j}=\min \left(\alpha_{i}, \alpha_{j}\right)$ and $s_{i j}=\min \left(\sigma_{i}, \sigma_{j}\right)$.

Lemma 9. $M_{I}(G)$ is a symmetric matrix.

Proof. We have $M_{I}(G)=\left(\left(r_{i j}, s_{i j}\right)\right)$ where $r_{i j}=\min \left(\alpha_{i}, \alpha_{j}\right)=\min \left(\alpha_{j}, \alpha_{i}\right)=$ $r_{j i}$ and $s_{i j}=\min \left(\sigma_{i}, \sigma_{j}\right)=\min \left(\sigma_{j}, \sigma_{i}\right)=s_{j i}$. Hence $M_{I}(G)$ is a symmetric matrix. 
Theorem 10. Let $G=(V, E, \mu, \gamma)$ be an intuitionistic fuzzy graph and $\alpha_{i}, \sigma_{j}$ are defined above. If $M_{I}(G)=\left(\left(r_{i j}, s_{i j}\right)\right)$ and if $r_{i j}=\min \left(\alpha_{i}, \alpha_{j}\right)$ and $s_{i j}=\min \left(\sigma_{i}, \sigma_{j}\right)$ then $\left(r_{i j}, s_{i j}\right)$ is an intuitionistic fuzzy set.

Proof. Let $r_{i j}=\min \left(\alpha_{i}, \alpha_{j}\right)$ and $s_{i j}=\min \left(\sigma_{i}, \sigma_{j}\right)$. If $r_{i j}=\alpha_{i}$ and $s_{i j}=\sigma_{i}$ then $0 \leq r_{i j}+s_{i j} \leq 1$. Now if $r_{i j}=\alpha_{i}$ and $s_{i j}=\sigma_{j}$ then $\alpha_{i} \leq \alpha_{j} \leq 1-\sigma_{j}$ $\Rightarrow \alpha_{i}+\alpha_{j} \leq 1$. Therefore $0 \leq \alpha_{i}+\alpha_{j} \leq 1$. Hence $\left(\alpha_{i}, \sigma_{j}\right)$ is an intuitionistic fuzzy set. Similarly we can prove the other cases also (if like $r_{i j}=\alpha_{j}, s_{i j}=\sigma_{i}$ and $r_{i j}=\alpha_{j}, s_{i j}=\sigma_{j}$ )

Definition 11. The energy of the In-Min intuitionistic fuzzy matrix of an intuitionistic fuzzy graph is defined as $E\left(M_{I}(G)\right)=\left(E\left(r_{i j}\right), E\left(s_{i j}\right)\right)$ where $E\left(r_{i j}\right)$ is the sum of the absolute of the eigen values of the matrix $r_{i j}$ and $E\left(s_{i j}\right)$ is the sum of the absolute of the eigen values of the matrix $s_{i j}$.

Definition 12. Let $G=(V, E, \mu, \gamma)$ be an intuitionistic fuzzy graph. For all vertex $i$, define $\theta_{i}=\max _{j} \mu_{i j}$ and $\phi_{i}=\min _{j} \gamma_{i j}$. Then it can be prove that $\left(\theta_{i}, \phi_{i}\right)$ is an intuitionistic fuzzy set on $V$.

Definition 13. Let $G=(V, E, \mu, \gamma)$ be an intuitionistic fuzzy graph. The Out-Min intuitionistic fuzzy matrix of an intuitionistic fuzzy graph is defined as $M_{O}(G)=\left(\left(u_{i j}, v_{i j}\right)\right)$ where $u_{i j}=\min \left(\theta_{i}, \theta_{j}\right)$ and $v_{i j}=\min \left(\phi_{i}, \phi_{j}\right)$ then it can be prove that $\left(u_{i j}, v_{i j}\right)$ is an intuitionistic fuzzy set. Also it can prove that $M_{O}(G)$ is a symmetric matrix.

Definition 14. The energy of the Out-Min intuitionistic fuzzy matrix of an intuitionistic fuzzy graph is defined as, $E\left(M_{O}(G)\right)=\left(E\left(u_{i j}\right), E\left(v_{i j}\right)\right)$ where $E\left(u_{i j}\right)$ is the sum of the absolute of the eigen values of the matrix $u_{i j}$ and $E\left(v_{i j}\right)$ is the sum of the absolute of the eigen values of the matrix $v_{i j}$.

\section{Example 15.}

For the graph in Fig.1, the In-Min intuitionistic fuzzy matrix is

$$
M_{I}\left(G_{1}\right)=\left(\begin{array}{cccc}
(0.9,0.1) & (0.8,0.1) & (0.5,0.1) & (0.6,0.1) \\
(0.8,0.1) & (0.8,0.1) & (0.5,0.1) & (0.6,0.1) \\
(0.5,0.1) & (0.5,0.1) & (0.5,0.1) & (0.5,0.1) \\
(0.6,0.1) & (0.6,0.1) & (0.5,0.1) & (0.6,0.3)
\end{array}\right)
$$




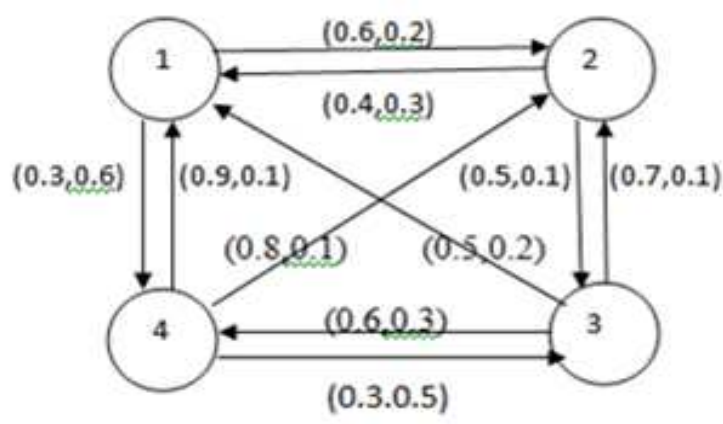

Figure 1: An Intuitionistic fuzzy graph G1

where $r_{i j}=\left(\begin{array}{cccc}0.9 & 0.8 & 0.5 & 0.6 \\ 0.8 & 0.8 & 0.5 & 0.6 \\ 0.5 & 0.5 & 0.5 & 0.5 \\ 0.6 & 0.6 & 0.5 & 0.6\end{array}\right)$ and $s_{i j}=\left(\begin{array}{cccc}0.1 & 0.1 & 0.1 & 0.1 \\ 0.1 & 0.1 & 0.1 & 0.1 \\ 0.1 & 0.1 & 0.1 & 0.1 \\ 0.1 & 0.1 & 0.1 & 0.3\end{array}\right)$

Here $E\left(M_{I}\left(G_{1}\right)\right)=(2.8000,0.6000)$. That is $E\left(M_{I}\left(r_{i j}\right)\right)>E\left(M_{I}\left(s_{i j}\right)\right)$, so we have $\beta=\max _{i, j} r_{i j}=0.9, \delta=\min _{i, j} s_{i j}=0.1, \tau=\frac{\beta}{\delta}=9$ and $\tau_{c}=\frac{1}{\lambda_{\max } r_{i j}}=$ $\frac{1}{2.4958}=0.4007$.

For the graph in Fig.1, the Out-Min intuitionistic fuzzy matrix is

$$
M_{O}\left(G_{1}\right)=\left(\begin{array}{cccc}
(0.6,0.2) & (0.5,0.1) & (0.6,0.1) & (0.6,0.1) \\
(0.5,0.1) & (0.5,0.1) & (0.5,0.1) & (0.5,0.1) \\
(0.6,0.1) & (0.5,0.1) & (0.7,0.1) & (0.7,0.1) \\
(0.6,0.1) & (0.5,0.1) & (0.7,0.1) & (0.9,0.1)
\end{array}\right)
$$

where $u_{i j}=\left(\begin{array}{cccc}0.6 & 0.5 & 0.6 & 0.6 \\ 0.5 & 0.5 & 0.5 & 0.5 \\ 0.6 & 0.5 & 0.7 & 0.7 \\ 0.6 & 0.5 & 0.7 & 0.9\end{array}\right)$ and $v_{i j}=\left(\begin{array}{cccc}0.2 & 0.1 & 0.1 & 0.1 \\ 0.1 & 0.1 & 0.1 & 0.1 \\ 0.1 & 0.1 & 0.1 & 0.1 \\ 0.1 & 0.1 & 0.1 & 0.1\end{array}\right)$

Here $E\left(M_{O}\left(G_{1}\right)\right)=(2.7000,0.5000)$. That is $E\left(M_{O}\left(u_{i j}\right)\right)>E\left(M_{O}\left(v_{i j}\right)\right)$, so we have $\beta=\max _{i, j} u_{i j}=0.9, \delta=\min _{i, j} v_{i j}=0.1, \tau=\frac{\beta}{\delta}=9$ and $\tau_{c}=\frac{1}{\lambda_{\max } u_{i j}}=$ $\frac{1}{2.4054}=0.4157$. 
Theorem 16. Let $G=(V, E, \mu, \gamma)$ be an intuitionistic fuzzy graph and if $r_{1}, r_{2}, \ldots . r_{n}$ are the eigen values of the matrix $r_{i j}$ then

$$
\sum_{i=1}^{n}\left(r_{i}\right)^{2}=\sum_{i=1}^{n}\left(r_{i i}\right)^{2}+2 \sum_{1 \leq i<j \leq n}\left(r_{i j}\right)^{2} .
$$

Proof. Sum of the square of the eigen values of $r_{i j}$ is equal to the trace of $\left(r_{i j}\right)^{2}$.

$$
\begin{gathered}
\sum_{i=1}^{n}\left(r_{i}\right)^{2}=\operatorname{trace}\left(r_{i j}\right)^{2} \\
=r_{11} r_{11}+r_{12} r_{21}+\ldots+r_{1 n} r_{n 1}+ \\
r_{21} r_{12}+r_{22} r_{22}+\ldots+r_{2 n} r_{n 2}+ \\
\ldots \ldots \ldots \ldots \ldots \ldots \ldots \ldots \ldots \ldots \ldots \ldots \ldots \ldots \ldots \ldots \ldots \\
r_{n 1} r_{1 n}+r_{n 2} r_{2 n}+\ldots+r_{n n} r_{n n} \\
\sum_{i=1}^{n}\left(r_{i}\right)^{2}=\sum_{i=1}^{n}\left(r_{i i}\right)^{2}+2 \sum_{1 \leq i<j \leq n}\left(r_{i j}\right)^{2} .
\end{gathered}
$$

Similarly we can prove if $s_{1}, s_{2}, \ldots . s_{n}$ are the eigen values of the matrix $s_{i j}$ then $\sum_{i=1}^{n}\left(s_{i}\right)^{2}=\sum_{i=1}^{n}\left(s_{i i}\right)^{2}+2 \sum_{1 \leq i<j<n}\left(s_{i j}\right)^{2}$ if $u_{1}, u_{2}, \ldots . u_{n}$ are the eigen values of the matrix $u_{i j}$ then $\sum_{i=1}^{n}\left(u_{i}\right)^{2}=\sum_{i=1}^{n}\left(u_{i i}\right)^{2}+2 \sum_{1 \leq i<j \leq n}\left(u_{i j}\right)^{2}$ if $v_{1}, v_{2}, \ldots v_{n}$ are the eigen values of the matrix $v_{i j}$ then $\sum_{i=1}^{n}\left(v_{i}\right)^{2}=\sum_{i=1}^{n}\left(v_{i i}\right)^{2}+$ $2 \sum_{1 \leq i<j \leq n}\left(v_{i j}\right)^{2}$.

Theorem 17. Let $G=(V, E, \mu, \gamma)$ be an intuitionistic fuzzy graph with $n$ vertices. Then

$$
\begin{gathered}
\sqrt{\sum_{i=1}^{n}\left(r_{i i}\right)^{2}+2 \sum_{1 \leq i<j \leq n}\left(r_{i j}\right)^{2}+n(n-1)|A|^{\frac{2}{n}}} \leq E\left(r_{i j}(G)\right) \\
\leq \sqrt{n\left(\sum_{i=1}^{n}\left(r_{i i}\right)^{2}+2 \sum_{1 \leq i<j \leq n}\left(r_{i j}\right)^{2}\right)}
\end{gathered}
$$

where $|A|$ is the determinant of $r_{i j}$. 
Proof. Upper bound. Cauchy Schwarz inequality is

$$
\left(\sum_{i=1}^{n} a_{i} b_{i}\right)^{2} \leq\left(\sum_{i=1}^{n} a_{i}^{2}\right)\left(\sum_{i=1}^{n} b_{i}^{2}\right) .
$$

If $a_{i}=1, b_{i}=\left|r_{i}\right|$ then

$$
\begin{aligned}
\left(\sum_{i=1}^{n}\left|r_{i}\right|\right)^{2} & \leq\left(\sum_{i=1}^{n} 1\right)\left(\sum_{i=1}^{n}\left(r_{i}\right)^{2}\right) \\
\left(E\left(r_{i j}(G)\right)\right)^{2} & \leq n\left(\sum_{i=1}^{n}\left(r_{i i}\right)^{2}+2 \sum_{1 \leq i<j \leq n}\left(r_{i j}\right)^{2}\right) \\
\Rightarrow E\left(r_{i j}(G)\right) & \leq \sqrt{n\left(\sum_{i=1}^{n}\left(r_{i i}\right)^{2}+2 \sum_{1 \leq i<j \leq n}\left(r_{i j}\right)^{2}\right)} .
\end{aligned}
$$

Lower bound.

$$
\begin{aligned}
\left(E\left(r_{i j}(G)\right)\right)^{2} & =\left(\sum_{i=1}^{n}\left|r_{i}\right|\right)^{2} \\
& =\sum_{i=1}^{n}\left|r_{i}\right|^{2}+2 \sum_{1 \leq i<j \leq n}\left|r_{i}\right|\left|r_{j}\right| \\
& =\left[\sum_{i=1}^{n}\left(r_{i i}\right)^{2}+2 \sum_{1 \leq i<j \leq n}\left(r_{i j}\right)^{2}\right]+2 \frac{n(n-1)}{2} A M\left\{\left|r_{i}\right|\left|r_{j}\right|\right\} .
\end{aligned}
$$

But, we know that $A M\left\{\left|r_{i}\right|\left|r_{j}\right|\right\} \geq G M\left\{\left|r_{i}\right|\left|r_{j}\right|\right\}, 1 \leq i<j \leq n$.

$$
\begin{aligned}
E\left(r_{i j}(G)\right) & \geq \sqrt{\sum_{i=1}^{n}\left(r_{i j}\right)^{2}+2 \sum_{1 \leq i<j \leq n}\left(r_{i j}\right)^{2}+n(n-1) G M\left\{\left|r_{i}\right|\left|r_{j}\right|\right\}} \\
G M\left\{\left|r_{i}\right|\left|r_{j}\right|\right\} & =\left(\prod_{i=1}^{n}\left|r_{i}\right|\left|r_{j}\right|\right)^{\frac{2}{n(n-1)}}=\left(\prod_{i=1}^{n}\left|r_{i}\right|^{(n-1))^{\frac{2}{n(n-1)}}}=|A|^{\frac{2}{n}}\right. \\
E\left(r_{i j}(G)\right) & \geq \sqrt{\sum_{i=1}^{n}\left(r_{i i}\right)^{2}+2 \sum_{1 \leq i<j \leq n}\left(r_{i j}\right)^{2}+n(n-1)|A|^{\frac{2}{n}}}
\end{aligned}
$$


From equations (1) and (2), we get

$$
\begin{gathered}
\sqrt{\sum_{i=1}^{n}\left(r_{i i}\right)^{2}+2 \sum_{1 \leq i<j \leq n}\left(r_{i j}\right)^{2}+n(n-1)|A|^{\frac{2}{n}}} \leq E\left(r_{i j}(G)\right) \\
\leq \sqrt{n\left(\sum_{i=1}^{n}\left(r_{i i}\right)^{2}+2 \sum_{1 \leq i<j \leq n}\left(r_{i j}\right)^{2}\right)}
\end{gathered}
$$

Similarly we can prove the bounds for the other cases.

Theorem 18. Let $G=(V, E, \mu, \gamma)$ be an intuitionistic fuzzy graph with $n$ vertices and if

$$
E\left(r_{i j}(G)\right) \leq 2 \sum_{i=1}^{n} \sum_{i=1}^{n} r_{i j} \quad, \quad E\left(s_{i j}(G)\right) \leq 2 \sum_{i=1}^{n} \sum_{i=1}^{n} s_{i j}
$$

then $E\left(r_{i j}(G)\right)+E\left(s_{i j}(G)\right) \leq 2 n^{2}$.

Proof. From the given statement we have

$$
\begin{aligned}
E\left(r_{i j}(G)\right) & \leq 2 \sum_{i=1}^{n} \sum_{i=1}^{n} r_{i j} \\
& \leq 2 \sum_{i=1}^{n} \sum_{i=1}^{n}\left(1-s_{i j}\right) \\
& =2 \sum_{i=1}^{n} \sum_{i=1}^{n} 1-2 \sum_{i=1}^{n} \sum_{i=1}^{n} s_{i j} \\
E\left(r_{i j}(G)\right)+E\left(s_{i j}(G)\right) & \leq 2 n^{2}-E\left(s_{i j}(G)\right)
\end{aligned}
$$

Hence the proof.

\section{Numerical Examples}

In this section, we illustrate the above concepts by taking the website http://www.pantechsolutions.net/. This website represented by a directed intuitionistic fuzzy graph. For this graph the spreading rate of virus is analyzed between incoming and outgoing links. 


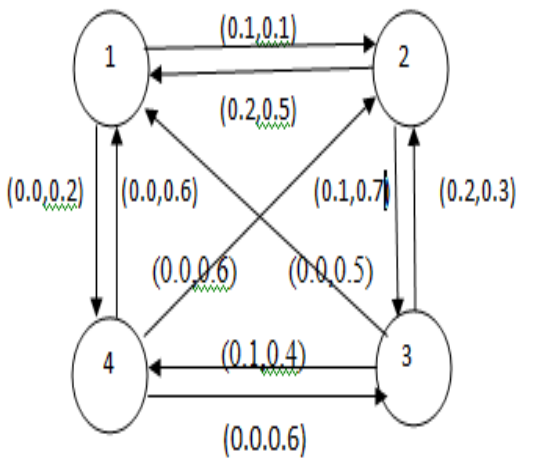

Figure 2: An Intuitionistic fuzzy graph G2

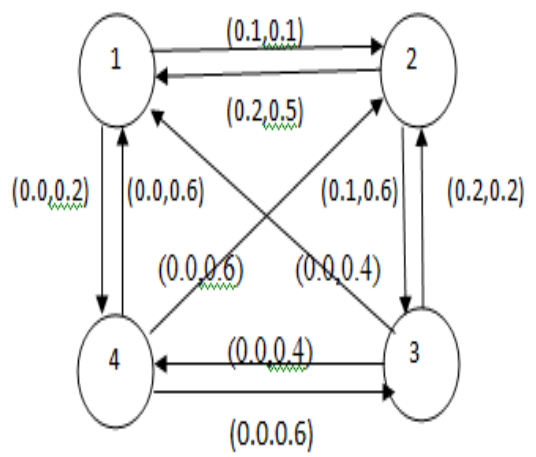

Figure 3: An Intuitionistic fuzzy graph G3

Example 19. In the above mentioned website, we considered the four links 1./microcontroller-boards, 2./log-in html, 3. / and 4./project kits for the period July 16, 2013 to August 15, 2013.

$$
\begin{array}{cl}
E\left(M_{I}\left(G_{2}\right)\right)=(0.6000,1.4000) & , \quad E\left(M_{O}\left(G_{2}\right)\right)=(0.5000,1.5000) \\
\beta=\min _{i, j} r_{i j}=0.1, \delta=\max _{i, j} s_{i j}=0.6 & , \quad \beta=\min _{i, j} u_{i j}=0, \delta=\max _{i, j} v_{i j}=0.6 \\
\tau=0.1667, \tau_{c}=\frac{1}{\lambda_{\max } s_{i j}}=0.8598 & , \quad \tau=0, \tau_{c}=\frac{1}{\lambda_{\max } v_{i j}}=0.7888 .
\end{array}
$$

Example 20. In the same website we consider the same four links for the period August 16, 2013 to September 15, 2013.

$$
E\left(M_{I}\left(G_{3}\right)\right)=(0.5000,1.3000) \quad, \quad E\left(M_{O}\left(G_{3}\right)\right)=(0.5000,1.4000)
$$




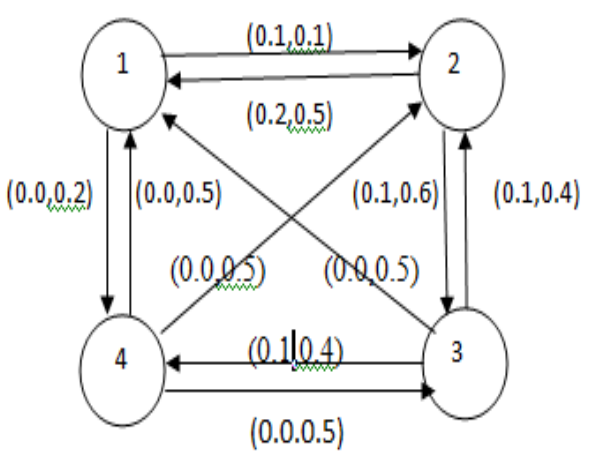

Figure 4: An Intuitionistic fuzzy graph G4

$$
\begin{gathered}
\beta=\min _{i, j} r_{i j}=0, \delta=\max _{i, j} s_{i j}=0.6 \quad, \quad \beta=\min _{i, j} u_{i j}=0, \delta=\max _{i, j} v_{i j}=0.6 \\
\tau=0, \tau_{c}=\frac{1}{\lambda_{\max } s_{i j}}=0.9624 \quad, \quad \tau=0, \tau_{c}=\frac{1}{\lambda_{\max } v_{i j}}=0.8598 .
\end{gathered}
$$

Example 21. In the same website, we consider the same four links for the period September 16, 2013 to October 15, 2013.

$$
\begin{gathered}
E\left(M_{I}\left(G_{4}\right)\right)=(0.5000,1.3000) \quad, \quad E\left(M_{O}\left(G_{4}\right)\right)=(0.4000,1.5000) \\
\beta=\min _{i, j} r_{i j}=0.1, \delta=\max _{i, j} s_{i j}=0.5 \quad, \quad \beta=\min _{i, j} u_{i j}=0, \delta=\max _{i, j} v_{i j}=0.5 \\
\tau=0.2, \tau_{c}=\frac{1}{\lambda_{\max } s_{i j}}=0.8954 \quad, \quad \tau=0, \tau_{c}=\frac{1}{\lambda_{\max } v_{i j}}=0.7332 .
\end{gathered}
$$

Example 22. In the same website, we consider the same four links for the period of October 16, 2013 to November 15, 2013.

$$
\begin{array}{cc}
E\left(M_{I}\left(G_{5}\right)\right)=(0.4000,1.3000) & , \quad E\left(M_{O}\left(G_{5}\right)\right)=(0.5000,1.5000) \\
\beta=\min _{i, j} r_{i j}=0, \delta=\max _{i, j} s_{i j}=0.1 & , \quad \beta=\min _{i, j} u_{i j}=0.1, \delta=\max _{i, j} v_{i j}=0.5 \\
\tau=0, \tau_{c}=\frac{1}{\lambda_{\max } s_{i j}}=0.9624 & , \quad \tau=0.2, \tau_{c}=\frac{1}{\lambda_{\max } v_{i j}}=0.7322 .
\end{array}
$$

The following table represents the comparison of the energies between incoming and outgoing links.

The following table represents the comparison of the spreading rate of virus between incoming and outgoing links. 


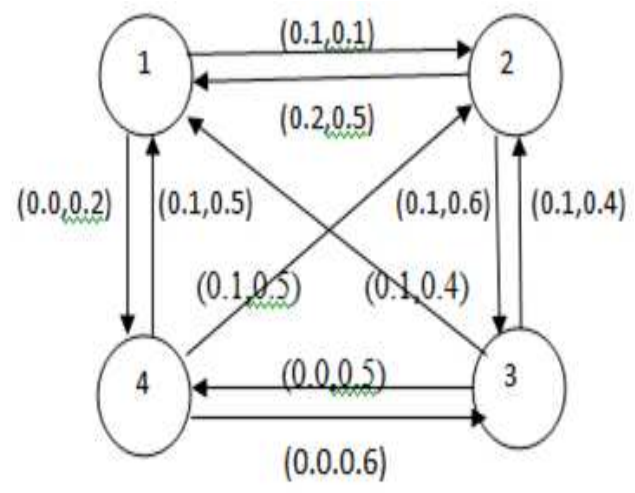

Figure 5: An Intuitionistic fuzzy graph G5

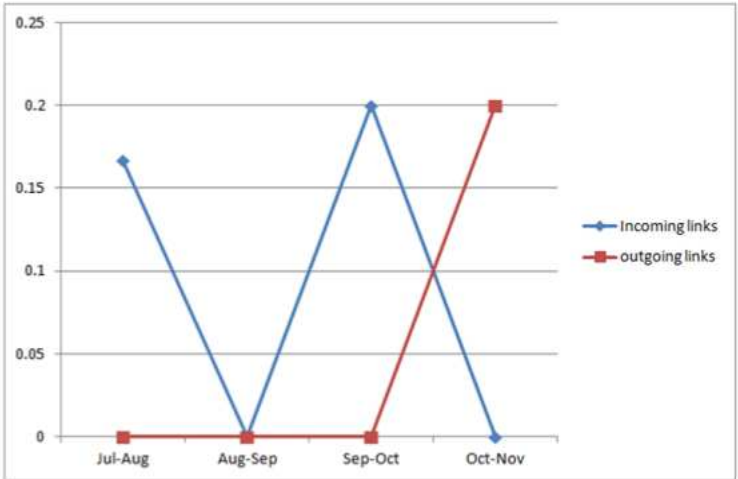

Figure 6: Spreading rate of virus between incoming and outgoing links 


\begin{tabular}{|c|c|c|c|}
\hline period & Energy of $A(G)$ & Energy of $M_{I}(G)$ & Energy of $M_{O}(G)$ \\
\hline Jul-Aug & $(0.4,2.1078)$ & $(0.6000,1.4000)$ & $(0.5000,1.5000)$ \\
\hline Aug-Sep & $(0.4,2.0068)$ & $(0.5000,1.3000)$ & $(0.5000,1.4000)$ \\
\hline Sep-Oct & $(0.3464,1.9129)$ & $(0.5000,1.3000)$ & $(0.4000,1.5000)$ \\
\hline Oct-Nov & $(0.3758,1.9879)$ & $(0.4000,1.3000)$ & $(0.5000,1.5000)$ \\
\hline
\end{tabular}

\begin{tabular}{|c|c|c|}
\hline period & $\tau$ on $M_{I}(G)$ & $\tau$ on $M_{O}(G)$ \\
\hline Jul-Aug & 0.1667 & 0 \\
\hline Aug-Sep & 0 & 0 \\
\hline Sep-Oct & 0.2 & 0 \\
\hline Oct-Nov & 0 & 0.2 \\
\hline
\end{tabular}

\section{Conclusion}

We conclude that the spreading rate of virus is minimum in outgoing links and it is maximum in incoming links which is shown in Fig.6. It is logically true that if number of visitors (i.e. incoming links) are maximum then the spreading rate of virus is maximum. It is verified through our real time example. Also we given the appropriate mathematical formulation for this real time example.

\section{Acknowledgments}

The author thank the management for their support towards the completion of this work.

\section{References}

[1] N. Anjali and Sunil Mathew, Energy of fuzzy graph, Annals of Fuzzy Mathematics and Informatics, 6, No. 3 (2013), 455-465.

[2] K. Atanassov, Intuitionistic Fuzzy sets: Theory and Applications, Springer-Verlag, Heidelberg (1999).

[3] N.T.J. Bailey, The Mathematical Theory of Infectious Diseases and its applications, Charlin Griffin, London (1975).

[4] D.J. Daley and J. Gani, Epidemic Modelling: An Introduction, Cambridge University Press, Cambridge (1999).

[5] G. Deepa, B. Praba and V.M. Chandrasekaran, Virus spread in an intuitionistic fuzzy network, International Journal of Applied Engineering Research, 9, No. 16 (2014), 55075515. 
[6] G. Deepa, B. Praba and V.M. Chandrasekaran, Max-Min intuitionistic fuzzy matrix of an intuitionistic fuzzy graph, International Journal of Pure and Applied Mathematics, 98, No. 3 (2015), 375-387.

[7] I. Gutman, The energy of a graph, Ber. Math - Statist. Sekt. Forschungsz. Graz, 103, (1978), 1-22.

[8] A. Kaufmann, Introduction a la Theorie des sous ensembles Flous, Masson et cie., Paris, 1 (1973).

[9] J.O. Kephart and S.R. White, Directed-Graph Epidemiological models of Computer Viruses, Proc. IEEE Computer Society Symposium on Research in Security and Privacy, USA (1991), 343-359.

[10] R. Pastor-Satorras and A. Vespignani, Epidemic spreading in scale - free networks, Phys. Rev. Lett., 86, No. 14 (2001), 3200-3203.

[11] B. Praba and R. Sujatha, Application of fuzzy Markov model for web-testing, Ganita Sandesh, 21, No. 2 (2007), 111-120.

[12] B. Praba, V.M. Chandrasekaran and G. Deepa, Energy of an intuitionistic fuzzy graph, Italian journal of pure and applied mathematics, No. 32 (2014), 431-444.

[13] P. Van Mieghem, Jasmina Omic and Robert Kooij, Virus spread in networks, IEEE/ACM Transactions on Networking, 17, No. 1 (2009), 1-14. 